\title{
SeleÇão de TraÇadores PaRa Estudos de EFICIÊNCIA das Aplicações de Produtos fitossanitários ${ }^{1}$
}

\author{
Selection of Tracer Dyes for Assessment Efficiency in Pesticide Applications
}

ALVES, G.S. ${ }^{2}$, CUNHA, J.P.A.R. ${ }^{2}$ e PALLADINI, L.A. ${ }^{3}$

\begin{abstract}
RESUMO - Para avaliar a deposição e as perdas de calda nas aplicações de produtos fitossanitários, na maioria dos casos são utilizados traçadores. Entretanto, não há consenso sobre qual é o traçador mais adequado para cada tipo de aplicação. O objetivo deste trabalho foi avaliar o uso de alguns compostos químicos empregados como traçadores, buscando selecionar os mais adequados aos estudos de deposição e perda de produtos fitossanitários. Os traçadores utilizados foram: azul brilhante, blankophor, fluoresceína sódica, rodamina B e sulfoflavina brilhante; as concentrações usadas foram: 1.000, 20, 15, 50 e 1.000 ppm, respectivamente. A quantificação do azul brilhante foi feita por espectrofotometria, e a dos demais, por espectrofluorimetria. Os filtros de excitação e emissão do fluorimetro variaram de acordo com o traçador. No espectrofotômetro, utilizou-se o comprimento de onda de $630 \mathrm{~nm}$. As características avaliadas nas soluções contendo os traçadores foram: tensão superficial, $\mathrm{pH}$, viscosidade, extração em alvos de papel filtrante e estabilidade à luz solar e à agitação. Os dados foram submetidos à análise de variância e, constatada diferença significativa, aplicou-se teste de médias e regressão. Entre as características físico-químicas, somente a viscosidade não foi influenciada pelos traçadores. A maior eficiência de extração em alvo de papel foi obtida pela sulfoflavina brilhante, que, juntamente com o azul brilhante, demonstrou ser o traçador mais estável à luz solar. O blankophor foi o traçador que teve menor estabilidade à agitação da calda. A sulfoflavina brilhante mostrou ser o traçador mais adequado aos estudos de deposição de calda e deriva nas aplicações de produtos fitossanitários. Entre os traçadores de baixo custo e maior disponibilidade no Brasil, o corante fluorescente rodamina B mostrou ser a melhor opção ao se utilizar como alvo papel filtrante.
\end{abstract}

Palavras-chave: deposição, perdas de calda, estabilidade, alvo artificial.

ABSTRACT - Tracers are used to evaluate pesticide spray deposition and loss; however, optimal tracers for specific applications have not yet been defined. Therefore, this study was aimed at evaluating various tracers and determining the best compound for assessing pesticide spray deposition and loss. We evaluated brilliant blue, blankophor, sodium fluorescein, rhodamine B and brilliant sulfoflavine at 1,000, 20, 15, 50, 1,000 ppm, respectively. Brilliant blue was evaluated by spectrophotometry (630 $\mathrm{nm}$ wavelength) and the other tracers by spectrofluorometry. The specific excitation and emission filters that were used in spectrofluorometry varied by tracer type. Surface tension, $\mathrm{pH}$, filter paper extraction, sunlight stability, and agitation stability were evaluated. The data were examined by variance analyses and the significant differences were submitted to average tests and regression. Viscosity was the only physical-chemical that was not affected by the tracer type. Brilliant sulfoflavine had the highest extraction efficiency, brilliant sulfoflavine and Brilliant Blue were the most stable in sunlight, and blankophor had the lowest agitation stability. Brilliant sulfoflavine was the most appropriate for pesticide spray deposition and drift assessment. Rhodamine $B$ showed to be the best option for filter paper targets among the low-cost tracers available in Brazil.

Keywords: spraying, losses, stability, artificial target.

Recebido para publicação em 10.7.2013 e aprovado em 22.7.2014.

UFU, Uberlândia-MG, Brasil; <guilhermeagro43@yahoo.com.br>; ${ }^{3}$ EPAGRI, Florianópolis-SC, Brasil.

Planta Daninha, Viçosa-MG, v. 32, n. 4, p. 861-870, 2014 


\section{INTRODUÇÃO}

A avaliação dos depósitos da calda aplicada em alvos naturais ou artificiais empregando-se traçadores é um ótimo método para compreender os diversos aspectos ligados à pulverização de produtos fitossanitários, em virtude da facilidade de sua visualização e remoção das folhas ou alvos coletores diretamente pela água (Marchi et al., 2005), dispensando-se assim componentes específicos para a extração e aparelhos com maior nivel de sofisticação. Caso essas pesquisas fossem realizadas utilizando-se os próprios ingredientes ativos, os custos seriam um dos fatores mais limitantes (Matuo, 1998).

Nesse sentido, os traçadores têm sido usados para avaliar a deposição e as perdas proporcionadas pela aplicação há vários anos (Fritz et al., 2011). Trata-se de substâncias adicionadas à calda para avaliar o desempenho dos pulverizadores, além de possibilitar mensurações qualitativas e quantitativas sobre os diversos destinos dos produtos fitossanitários no ambiente aplicados por um equipamento de pulverização. Trazem como vantagem geral - em relação à detecção empregando-se o próprio ingrediente ativo - maior facilidade e menores custos das análises químicas e risco de contaminação ao usuário. Contudo, quando a calda é preparada apenas adicionando-se os traçadores, não incluindo os produtos, não há real representação da composição da calda, o que pode interferir nos resultados.

Ao usar os traçadores, o primeiro passo importante refere-se à sua escolha, em que se deve levar em consideração, sobretudo, a estabilidade à luz solar, o nível de detecção, a toxicidade, a persistência e a solubilidade (Palladini et al., 2005), além da taxa de recuperação e a influência sobre as propriedades físico-químicas da calda (Pergher, 2001). É importante analisar a estabilidade dos traçadores à luz solar, pelo fato de a maioria dos experimentos relacionados à deposição e à deriva ser realizada no campo.

Entre os traçadores fluorescentes, merece destaque a sulfoflavina brilhante, por apresentar boas características de estabilidade à luz solar (Brusselman et al., 2004). Além disso, apresenta possibilidade de detecção em quantidades bastante reduzidas, com limite de detecção de 0,005 a 0,0005 $\mu \mathrm{g} \mathrm{cm}^{-2}$ (Nuyttens et al., 2007). No entanto, seu custo é elevado e tem baixa disponibilidade em alguns mercados, como observado no Brasil. Em estudos de aplicação aérea, por exemplo, em que se tem a necessidade de trabalhar com a calda bastante concentrada, o custo praticamente inviabiliza seu uso. Assim, faz-se necessário avaliar outros tipos de traçadores fluorescentes, com menor custo e melhor acessibilidade.

Hayden et al. (1990) foram um dos pioneiros no trabalho com corantes alimentícios, concluindo que eles podem ser utilizados nas pesquisas de avaliações de depósitos em plantas, por ser um método preciso e barato. Em seguida, passaram a ser amplamente utilizados em estudos de deposição de calda em alvos naturais, sendo muito empregado o azul brilhante, por possuir boa estabilidade à luz solar (Pinto et al., 2007). Contudo, por possuir nível de detecção menor do que o de outros traçadores, torna-se dificil utilizá-lo em estudos de deriva, em que se requerem determinações de quantidades bastante diminutas.

Tendo em vista que não há consenso sobre o traçador ideal, a pequena disponibilidade e o alto custo em alguns mercados desses produtos empregados com frequência na literatura internacional, objetivou-se avaliar o uso de alguns compostos químicos usados como traçadores, buscando selecionar os mais adequados aos estudos de deposição e perdas de calda de produtos fitossanitários.

\section{MATERIAL E MÉTODOS}

Os traçadores utilizados foram: azul brilhante - AB (Duas Rodas, Jaraguá do Sul, Brasil); blankophor BA 267\% - BKP (Lanxess, Leverkusen, Alemanha); fluoresceína sódica - FSS (Synth, Diadema, Brasil); rodamina B RDM (Synth, Diadema, Brasil); e sulfoflavina brilhante - BSF (Biovalley, Marne La Vallee, França).

A quantificação dos traçadores fluorescentes foi feita por fluorimetria, com auxilio de um fluorimetro com lâmpada de halogênio (Thermo Scientific, FM109515). Na Tabela 1 são mostrados os filtros de emissão e excitação utilizados para detecção por fluorescência. Para o corante azul brilhante, a quantificação foi feita por espectrofotometria, utilizando-se um 
Tabela 1 - Filtros de excitação e emissão de onda utilizados no fluorímetro para detecção dos traçadores fluorescentes

\begin{tabular}{|l|c|c|l|}
\hline \multirow{2}{*}{\multicolumn{1}{|c|}{ Traçador }} & \multicolumn{2}{c|}{ Filtro } & \multirow{2}{*}{ Referência } \\
\cline { 2 - 3 } & Excitação & Emissão & \\
\hline Rodamina B & NB540 & SC585 & Chaim et al. (2003) \\
\hline Blankophor & NB360 & SC415 & Villalba et al. (2011) \\
\hline Fluoresceína Na & NB490 & SC515 & Zhu et al. (2005) \\
\hline Sulfoflavina brilhante & NB390 & SC500 & Magdalena (2004) \\
\hline
\end{tabular}

espectofotômetro com lâmpada de tungstêniohalogênio (Biospectro, SP22). Utilizou-se o comprimento de onda de $630 \mathrm{~nm}$, faixa de detecção do azul, de acordo com a metodologia empregada por Palladini et al. (2005).

As concentrações iniciais teóricas das soluções contendo os traçadores $\mathrm{AB}, \mathrm{BKP}, \mathrm{BSF}$, FSS e RDM foram: 1.000 (De Marchi et al., 2009), 20 (Villalba et al., 2011), 1.000 (De Schampheleire et al., 2009), 15 (Zhu et al., 2005) e 50 ppm (Chaim et al., 2003), respectivamente. Convém ressaltar que foram utilizadas essas concentrações devido ao fato de serem as mesmas usadas pelos autores nas aplicações em campo.

A calibração do fluorímetro foi feita utilizando-se soluções com concentrações conhecidas, sendo: 0,1, 4,0, 0,1 e 0,2 ppm, para BKP, BSF, FSS e RDM, respectivamente. Para o azul brilhante, fez-se uma curva de calibração, conforme consta na Figura 1.

\section{Caracteristicas físico-quimicas de soluções aquosas de traçadores}

As características avaliadas foram: tensão superficial, $\mathrm{pH}$ e viscosidade. Os ensaios

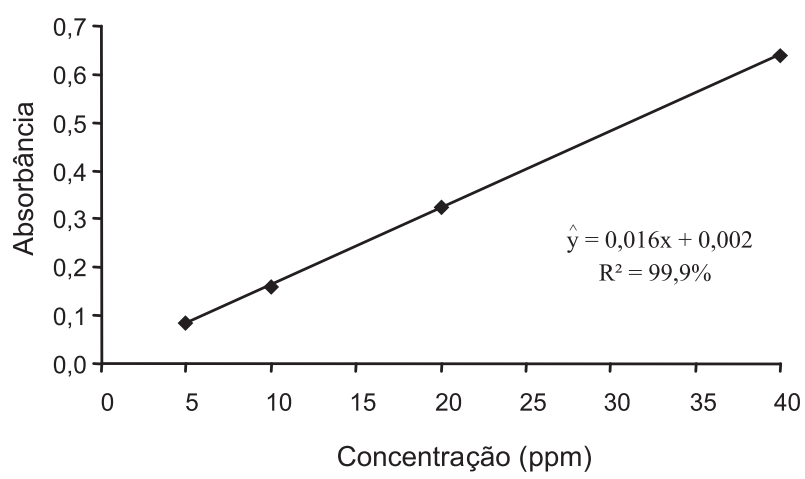

Figura 1 - Curva de calibração para detecção do azul brilhante no espectrofotômetro. foram feitos em delineamento inteiramente casualizado (DIC) com quatro repetições. Como referência, avaliaram-se também as características da água destilada. As soluções de cada repetição foram preparadas em balões volumétricos de $100 \mathrm{~mL}$. Seguiram-se as metodologias empregadas por Cunha \& Alves (2010).

Para determinação da tensão superficial, foi utilizado um tensiômetro de bancada com anel de platina (Kruss, K6). O pH foi medido por um peagômetro portátil (Hanna, HI98139), que foi previamente calibrado utilizando-se soluções-padrão de pH 7,0 e 4,0.

A viscosidade foi determinada por meio de um viscosímetro rotativo microprocessado (Quimis, 860M21). Utilizou-se o rotor zero na rotação de $60 \mathrm{rpm}$.

\section{Eficiência de extração em alvo artificial}

A eficiência de extração foi determinada conforme método utilizado por Rezende (2011). Como alvo, foram utilizados papéis filtrantes com pH neutro e gramatura de $65 \mathrm{~g} \mathrm{~m}^{-2}$ (Jprolab, São José dos Pinhais, Brasil), com dimensões de $3 \times 3 \mathrm{~cm}$, por serem muito utilizados em estudos de deriva, onde é necessário captar o produto perdido para fora da área-alvo. O ensaio foi feito em DIC, com quatro repetições, em esquema fatorial $5 \times 5$, sendo o primeiro fator referente aos traçadores e o segundo, ao tempo entre a distribuição e o início do processo de extração deles do papel.

Individualmente, os papéis foram colocados em placas de Petri e, com o auxílio de uma pipeta automática com capacidade de $1.000 \mu \mathrm{L}$, distribuíram-se sobre eles $50 \mu \mathrm{L}$ da solução contendo os traçadores. Após a distribuição, esperou-se 30, 60, 120, 240 e 360 minutos para se fazer a extração. Após atingir o tempo, os alvos foram transferidos para copos plásticos com tampas, em que foram adicionados $15 \mathrm{~mL}$ de água destilada, exceto para a rodamina B. Nesse caso, foi utilizado o mesmo volume, porém de uma solução de água destilada e Tween 80 (polioxietilensorbitano mono-oleato) a $0,2 \%$, seguindo-se a metodologia usada por Scramin et al. (2002).

Em seguida, exceto para o blankophor, foi feita uma agitação durante 15 minutos

Planta Daninha, Viçosa-MG, v. 32, n. 4, p. 861-870, 2014 
em uma mesa agitadora pendular (Tecnal, TE-240/I), seguindo as metodologias usadas por Scramin et al. (2002) para a rodamina B; Rezende (2011), para a fluoresceína sódica e azul brilhante; e De Schampheleire et al. (2009), para a sulfoflavina. Padronizou-se a rotação em $120 \mathrm{rpm}$, por ser a mesma usada por Scramim et al. (2002) e por possibilitar fazer a extração de todos os traçadores simultaneamente. Para o BKP, depois da adição de água, retiraram-se os papéis após leve agitação manual, conforme Villalba et al. (2011).

Adicionalmente, o mesmo foi feito em placas de Petri sem os alvos, a fim de obter a concentração real inicial. De posse desses dados, calculou-se a porcentagem de recuperação dos traçadores em relação à concentração inicial real, segundo a Equação 1:

$$
\% \operatorname{Re} c=\frac{C_{\text {inst }}}{C_{\text {real }}} x 100
$$

em que \%Rec - porcentagem de recuperação; $\mathrm{C}_{\text {inst }}$ - concentração do traçador obtida após a extração (ppm); e $\mathrm{C}_{\text {real }}$ - concentração real inicial do traçador após a diluição (ppm).

\section{Estabilidade à luz solar}

Para avaliar o comportamento dos traçadores quando expostos à luz solar, foram colocados $100 \mathrm{~mL}$ de solução de cada traçador dentro de placas de Petri, com fundo e bordas totalmente translúcidos, dispostas sobre um solo sem cobertura vegetal. As placas não foram tampadas, para não haver interferência no processo de evaporação da água.

O ensaio foi realizado em delineamento de blocos casualizados (DBC) com quatro repetições, em esquema de parcelas subdivididas no tempo $5 \times 6$, sendo o fator de parcela referente aos traçadores e o de subparcela, aos tempos de exposição solar: 15, 30, 45, 60, 90 e 120 minutos. Após atingir o tempo, retiraramse da placa de Petri $10 \mathrm{~mL}$ da solução, que foram transferidos para copos plásticos acondicionados em caixas de isopor. O período de exposição foi das 9 h30 às 11 h30, horário em que não havia nuvens no céu. Segundo dados do Instituto Nacional de Meteorologia (INMET, 2013), a radiação solar emitida durante a condução do ensaio foi de $6,862 \mathrm{MJ} \mathrm{m}^{-2}$. Para obter a concentração inicial real das soluções, foram feitas leituras de soluções que não foram expostas ao sol. Em laboratório, as amostras foram diluídas, adicionando-se 40, 50, 40, 65 e $40 \mu \mathrm{L}$ das soluções contendo AB, BKP, BSF, FSS e RDM, respectivamente, em $10 \mathrm{~mL}$ de água destilada, para que fosse feita a leitura direta no aparelho. Com isso, pôde-se calcular a porcentagem de recuperação dos traçadores por meio da Equação 1.

\section{Estabilidade à agitação}

O intuito desta avaliação foi simular a agitação proporcionada pelo tanque do pulverizador. O ensaio foi realizado em DIC com quatro repetições, em esquema fatorial $5 \times 4$, sendo o primeiro fator referente aos traçadores e o segundo, aos tempos de agitação.

Foram adicionados $10 \mathrm{~mL}$ de solução com cada traçador em recipientes plásticos com capacidade de $100 \mathrm{~mL}$. Os frascos foram lacrados e submetidos à agitação durante 10 , 20, 30 e 60 minutos, na rotação de $150 \mathrm{rpm}$. Para isso, foi utilizada uma mesa agitadora pendular (Tecnal, TE-240/I). Para obter a concentração inicial real das soluções, foram feitas leituras das amostras não submetidas à agitação. Ao término da agitação, as soluções foram diluídas, adicionando-se 40, 50, 40, 65 e $40 \mu \mathrm{L}$ das soluções contendo AB, BKP, BSF, FSS e RDM, respectivamente, em $10 \mathrm{~mL}$ de água destilada, para que fosse feita a leitura direta no aparelho. Com isso, pôde-se calcular a porcentagem de recuperação dos traçadores por meio da Equação 1.

\section{Análises estatísticas}

Inicialmente, foram testadas as pressuposições dos dados utilizando-se o programa estatístico SPSS 17.0 (SPSS, 2008). Nos ensaios conduzidos em DIC, aplicaram-se os testes de Levene, para verificar a homogeneidade de variâncias, e Kolmogorov-Smirnov (para número de observações maior que 50) ou Shapiro-Wilk (para número de observações menor do que 50), para testar a normalidade dos resíduos. No ensaio conduzido em DBC, além destes, aplicou-se também o teste de Tukey para aditividade de blocos. Nos casos em que as pressuposições não foram atendidas a 0,01 de significância, os dados foram 
transformados em raiz quadrada de $\mathrm{x}$ e submetidos a nova análise. Somente quando a transformação corrigiu ou melhorou pelo menos um dos critérios, sem prejudicar os demais, usaram-se os dados transformados para elaborar a análise de variância. Do contrário, foram utilizados os dados originais.

Após analisar as pressuposições, os dados foram submetidos à análise de variância pelo programa estatístico Sisvar 5.3 (Ferreira, 2008) em todos os ensaios, exceto para as características físico-químicas das soluções, em que foi utilizado o programa Assistat 7.6 beta (Silva \& Azevedo, 2009). Em todas as análises, adotou-se a significância de 0,05 .

Pelo Sisvar, os tratamentos qualitativos foram comparados entre si pelo teste de Tukey, enquanto os quantitativos foram submetidos à análise de regressão. As curvas foram elaboradas pelo programa Table Curve 2D 5.01 (SPSS, 2000).

Pelo Assistat, a tensão superficial, o pH e a viscosidade das soluções dos traçadores foram comparados com a testemunha (água destilada), utilizando-se o teste de Dunnett.

\section{RESULTADOS E DISCUSSÃO}

Somente para tensão superficial, $\mathrm{pH}$ e viscosidade, os dados originais mostraram resíduos com distribuição normal e variâncias homogêneas. Quanto às demais características, mesmo após a transformação, no geral, as pressuposições não foram atendidas a 0,01 de significância. A transformação dos dados referentes à agitação melhorou a homogeneidade das variâncias e a normalidade dos resíduos. Assim, a análise de variância foi elaborada usando-se os dados originais para todas as características, exceto para agitação, em que foram utilizados os dados transformados.

Como pode ser observado na Tabela 2, somente as soluções com RDM e BSF apresentaram tensão superficial menor do que a da água destilada, que foi de $72,6 \mathrm{mN} \mathrm{m}^{-1}$; a diferença foi de 6,43 para a RDM e de $1,38 \mathrm{mN} \mathrm{m}^{-1}$ para a BSF. Entre os traçadores, a RDM também apresentou a menor tensão $\left(66,17 \mathrm{mN} \mathrm{m}^{-1}\right)$. No entanto, vale ressaltar que essa redução não é tão expressiva como a resultante do uso de adjuvantes redutores de tensão superficial. Cunha \& Alves (2009), por exemplo, encontraram valores de tensão superficial de $43,2 \mathrm{mN} \mathrm{m}^{-1}$ com a adição do surfatante nonil fenol etanol+sal sódico dodecil benzeno sulfônico à água. Além disso, a detecção dessas diferenças se dá provavelmente pelo baixo coeficiente de variação dos dados $(0,66 \%)$. O BKP, a FSS e o AB não modificaram a tensão superficial da água e não diferenciaram entre si.

Todas as soluções contendo os traçadores apresentaram $\mathrm{pH}$ menor do que o da água destilada. Contudo, essa redução foi mais expressiva para a BSF, com $\mathrm{pH}$ de 4,31. A menor redução foi observada para a FSS, com pH de 6,06 . Por outro lado, os traçadores não interferiram na viscosidade da água destilada, não havendo diferenças entre eles, resultando em valores entre 1,17 e 1,32 $\mathrm{mPa}$ s.

Assim, os traçadores avaliados pouco interferiram nas características físico-químicas das soluções, com exceção para o pH utilizando-se a BSF. No entanto, como na literatura existem trabalhos empregando várias concentrações para um mesmo traçador, novos estudos são necessários, caso sejam usadas concentrações diferentes das empregadas neste trabalho.

Tabela 2 - Características físico-químicas de soluções de traçadores usados em estudos de eficiência das aplicações de produtos fitossanitários

\begin{tabular}{|l|c|c|c|}
\hline \multicolumn{1}{|c|}{ Traçador } & $\begin{array}{c}\text { Tensão Superficial } \\
\left(\mathrm{mN} \mathrm{m}^{-1}\right)\end{array}$ & $\mathrm{pH}$ & $\begin{array}{c}\text { Viscosidade } \\
(\mathrm{mPa} \mathrm{s})\end{array}$ \\
\hline Rodamina B & $66,17 \mathrm{c}^{+}$ & $5,51 \mathrm{c}^{+}$ & $1,29 \mathrm{a}$ \\
\hline Blankophor & $73,12 \mathrm{a}$ & $5,96 \mathrm{ab}^{+}$ & $1,32 \mathrm{a}$ \\
\hline Sulfoflavina & $71,22 \mathrm{~b}^{+}$ & $4,31 \mathrm{~d}^{+}$ & $1,31 \mathrm{a}$ \\
\hline Fluoresceína Na & $72,77 \mathrm{a}$ & $6,06 \mathrm{a}^{+}$ & $1,19 \mathrm{a}$ \\
\hline Azul brilhante & $72,57 \mathrm{a}$ & $5,67 \mathrm{bc}^{+}$ & $1,17 \mathrm{a}$ \\
\hline Água destilada & 72,60 & 6,31 & 1,25 \\
\hline CV (\%) & 0,66 & 2,71 & 5,95 \\
\hline DMS Dunnett & 0,96 & 0,30 & 0,16 \\
\hline DMS Tukey & 1,15 & 0,32 & 0,17 \\
\hline$F_{\text {trạxtest }}$ & $115,719^{* *}$ & $109,618^{* *}$ & $2,865^{*}$ \\
\hline $\mathrm{F}_{\text {trac }}$ & $119,659^{* *}$ & $89,293^{* *}$ & $3,480^{*}$ \\
\hline
\end{tabular}

Médias seguidas por letras distintas nas colunas diferem entre si pelo teste de Tukey. Médias seguidas por ${ }^{+}$diferem da testemunha pelo teste de Dunnett. CV: coeficiente de variação. DMS: diferença mínima significativa. $\mathrm{F}_{\text {trac }}$ e $\mathrm{F}_{\text {tracxtest }}$ : valores do $\mathrm{F}$ calculado para traçador e interação entre traçador e testemunha, respectivamente. ** significativo a $0,01{ }^{*}$ significativo a 0,05 . 
Schleier et al. (2010), avaliando o efeito de traçadores fluorescentes no espectro de gotas e nas características das caldas de aplicação, concluíram que os traçadores óleo solúvel Tinopal OB e a fluoresceína também não afetaram significativamente as propriedades de densidade e viscosidade da calda, tampouco o diâmetro da mediana volumétrica do espectro de gotas. Esses autores sugerem ainda que, provavelmente, os traçadores têm pouca influência sobre o movimento da calda aplicada no ambiente, concordando com os resultados deste trabalho.

Na Tabela 3 é mostrada a eficiência de extração de traçadores em alvos artificiais de papel. Nota-se que a maior porcentagem de recuperação, em todos os tempos de contato, foi obtida para a BSF, que teve o valor mais próximo de $100 \%$, considerado como ideal. A FSS teve recuperação similar à da BSF somente nos dois primeiros tempos de contato. Outro traçador que demonstrou ter boa recuperação, porém menor do que a de BSF, foi a RDM, resultando em eficiência de extração acima de $84 \%$. Os piores valores foram obtidos pelos traçadores AB e BKP, sendo abaixo de 52 e 44\%, respectivamente. Assim, estes traçadores devem ser evitados quando se deseja trabalhar com esse tipo de alvo, por subestimarem a quantificação dos depósitos, influenciando negativamente na análise. Vale ressaltar que, segundo Palladini et al. (2005), o AB apresentou bom comportamento como traçador em extração diretamente de folhas.

A qualidade da extração não foi influenciada pelo tempo de contato em que os traçadores foram mantidos com os alvos até o início da extração. Dessa forma, nenhum modelo de regressão foi significativo a 0,05 , o que indica que a eficiência obtida no menor tempo de contato (30 $\mathrm{min})$ foi a mesma obtida no tempo máximo (360 min). Isso significa que a extração dos traçadores não necessariamente deve ser feita instantaneamente após a aplicação. Pode-se esperar um tempo de até seis horas para se fazer a extração sem que a recuperação dos traçadores no papel seja prejudicada. Caso o intervalo entre a aplicação e a extração seja maior do que seis horas, novos estudos são necessários, a fim de verificar se os resultados teriam o mesmo comportamento.

Planta Daninha, Viçosa-MG, v. 32, n. 4, p. 861-870, 2014
A Tabela 4 refere-se à porcentagem de recuperação dos traçadores expostos por vários tempos à luz solar. $\mathrm{O} A \mathrm{~A}$ mostrou ser o mais estável durante todo o tempo de exposição, apresentando valores entre 99,2 e 106,5\%. De maneira geral, a RDM demonstrou ser o segundo traçador fluorescente mais estável à luz solar, resultando em porcentagem de recuperação superior a $91 \%$ até 60 minutos de exposição. A BSF também apresentou boa estabilidade, igualando-se ao corante $\mathrm{AB}$ nos últimos tempos de exposição (90 e $120 \mathrm{~min}$ ). Expondo-se a FSS por um tempo de $15 \mathrm{~min}$, houve queda de aproximadamente $50 \%$, demonstrando ter a maior instabilidade à luz solar entre os traçadores avaliados, mesmo quando a exposição foi por tempo reduzido.

A RDM e o BKP demonstraram ter decréscimo linear, e a FSS, exponencial, na porcentagem de recuperação à medida que o tempo de exposição aumentou (Figura 2). Para a RDM, a cada minuto de exposição à luz solar, esperase redução de $0,15 \%$ na porcentagem de recuperação, chegando ao valor mínimo de $79 \%$ no tempo de 120 min. Para o BKP, essa redução estimada é de $0,31 \%$, chegando à porcentagem mínima de 55\%. No caso específico da FSS, observou-se que a maior taxa de decréscimo ocorreu antes dos 60 minutos iniciais, tendo porcentagem de recuperação estabilizada próximo dos $6 \%$ no término da exposição - considerado um valor extremamente baixo. Suhogusoff et al. (2005) concluíram que esse mesmo traçador, na concentração de $70 \mathrm{mg} \mathrm{L}^{-1}$, teve perda de massa de até $20 \%$ quando exposto à luz do ambiente (artificial e natural), corroborando os resultados deste trabalho.

Por outro lado, para Gouzie et al. (2010), a RDM possui elevada estabilidade à luz solar, tendo baixa degradação durante o período investigativo, o que está de acordo com este trabalho, desde que a exposição não seja superior a 60 minutos.

Ainda na Tabela 4, nota-se que para os traçadores $\mathrm{AB}$ e $\mathrm{BSF}$ nenhum modelo de regressão foi significativo, indicando que não houve diferença entre os dados presentes no intervalo de 15 a 120 minutos de exposição, o que reforça a ideia de que esses traçadores foram os mais estáveis. Pinto et al. (2007), avaliando a estabilidade de alguns corantes à luz solar, concluíram que o AB é estável por um 
Tabela 3 - Eficiência de extração (\%) de traçadores em alvos de papel filtrante, variando-se o intervalo de tempo entre a aplicação e o início da extração

\begin{tabular}{|c|c|c|c|c|c|}
\hline \multirow{2}{*}{ Tempo (min) } & \multicolumn{5}{|c|}{ Traçador $^{1 /}$} \\
\hline & RDM & BKP & BSF & FSS & $\mathrm{AB}$ \\
\hline 30 & $89,9 \mathrm{~b}$ & $37,4 \mathrm{c}$ & $99,6 \mathrm{a}$ & $91,8 \mathrm{ab}$ & $37,3 \mathrm{c}$ \\
\hline 60 & $84,7 \mathrm{~b}$ & $42,9 \mathrm{c}$ & $97,5 \mathrm{a}$ & $94,1 \mathrm{a}$ & $48,7 \mathrm{c}$ \\
\hline 120 & $88,7 \mathrm{~b}$ & $43,7 \mathrm{c}$ & $105,7 \mathrm{a}$ & $86,1 \mathrm{~b}$ & $50,4 \mathrm{c}$ \\
\hline 240 & $88,2 \mathrm{~b}$ & $42,4 d$ & $103,4 \mathrm{a}$ & $84,4 \mathrm{~b}$ & $51,3 \mathrm{c}$ \\
\hline 360 & $86,9 \mathrm{~b}$ & $43,8 \mathrm{c}$ & $103,2 \mathrm{a}$ & $87,3 \mathrm{~b}$ & $46,0 \mathrm{c}$ \\
\hline \multicolumn{3}{|c|}{$\mathrm{DMS}=7,9$} & \multirow{2}{*}{\multicolumn{3}{|c|}{$\begin{array}{c}\mathrm{F}_{\text {trac }}=931,104^{* *} ; \mathrm{F}_{\text {temp }}=2,380 \mathrm{~ns} \\
\mathrm{~F}_{\text {inter }}=3,318^{* *}\end{array}$}} \\
\hline \multicolumn{3}{|c|}{$\mathrm{CV}=5,41 \%$} & & & \\
\hline
\end{tabular}

Médias seguidas por letras distintas nas linhas diferem entre si pelo teste de Tukey a 0,05 de significância. DMS: diferença mínima significativa. CV: coeficiente de variação. $\mathrm{F}_{\text {trạ̣ }}, \mathrm{F}_{\text {temp }}, \mathrm{F}_{\text {inter }}$ - valores de $\mathrm{F}$ calculado para os fatores traçador, tempo e interação entre os fatores, respectivamente. ${ }^{* *}$ significativo a 0,01 ; ${ }^{*}$ significativo a 0,05 ; ns: não significativo. ${ }^{1 /}$ RDM: rodamina B; BKP: blankophor; BSF: sulfoflavina; FSS: fluoresceína sódica; AB: azul brilhante.

Tabela 4 - Porcentagem de recuperação de traçadores submetidos a diferentes tempos de exposição à luz solar

\begin{tabular}{|c|c|c|c|c|c|}
\hline \multirow{2}{*}{$\begin{array}{c}\text { Tempo } \\
(\mathrm{min})\end{array}$} & RDM & BKP & Braçador ${ }^{1 /}$ & FSS & AB \\
\cline { 2 - 5 } & $93,9 \mathrm{c}$ & $87,4 \mathrm{~d}$ & $105,2 \mathrm{a}$ & $50,1 \mathrm{e}$ & $99,2 \mathrm{~b}$ \\
\hline 15 & $94,2 \mathrm{~b}$ & $83,4 \mathrm{c}$ & $103,9 \mathrm{a}$ & $38,9 \mathrm{~d}$ & $100,8 \mathrm{a}$ \\
\hline 30 & $91,3 \mathrm{~b}$ & $83,0 \mathrm{c}$ & $106,1 \mathrm{a}$ & $23,2 \mathrm{~d}$ & $101,8 \mathrm{a}$ \\
\hline 45 & $91,1 \mathrm{c}$ & $75,4 \mathrm{~d}$ & $109,1 \mathrm{a}$ & $18,1 \mathrm{e}$ & $101,6 \mathrm{~b}$ \\
\hline 60 & $83,1 \mathrm{~b}$ & $62,5 \mathrm{c}$ & $104,4 \mathrm{a}$ & $7,4 \mathrm{~d}$ & $105,6 \mathrm{a}$ \\
\hline 90 & $79,0 \mathrm{~b}$ & $56,3 \mathrm{c}$ & $106,0 \mathrm{a}$ & $5,8 \mathrm{c}$ & $106,5 \mathrm{a}$ \\
\hline 120 & $\mathrm{DMS}=4,5$ & \multirow{2}{*}{$\mathrm{F}_{\text {traç }}=2403,845^{* *} ; \mathrm{F}_{\text {temp }}=190,144^{* *} ; \mathrm{F}_{\text {inter }}=71,249^{* *}$} \\
\hline
\end{tabular}

Médias seguidas por letras distintas nas linhas diferem entre si pelo teste de Tukey a 0,05 de significância. DMS: diferença mínima significativa. CV: coeficiente de variação. $\mathrm{F}_{\text {trac }}, \mathrm{F}_{\text {temp }}, \mathrm{F}_{\text {inter }}$ - valores de $\mathrm{F}$ calculado para os fatores traçador, tempo e interação entre os fatores, respectivamente. ${ }^{* *}$ significativo a 0,$01 ;{ }^{*}$ significativo a $0,05 .{ }^{1 /}$ RDM: rodamina B; BSF: sulfoflavina; FSS: fluoresceína sódica; AB: azul brilhante.
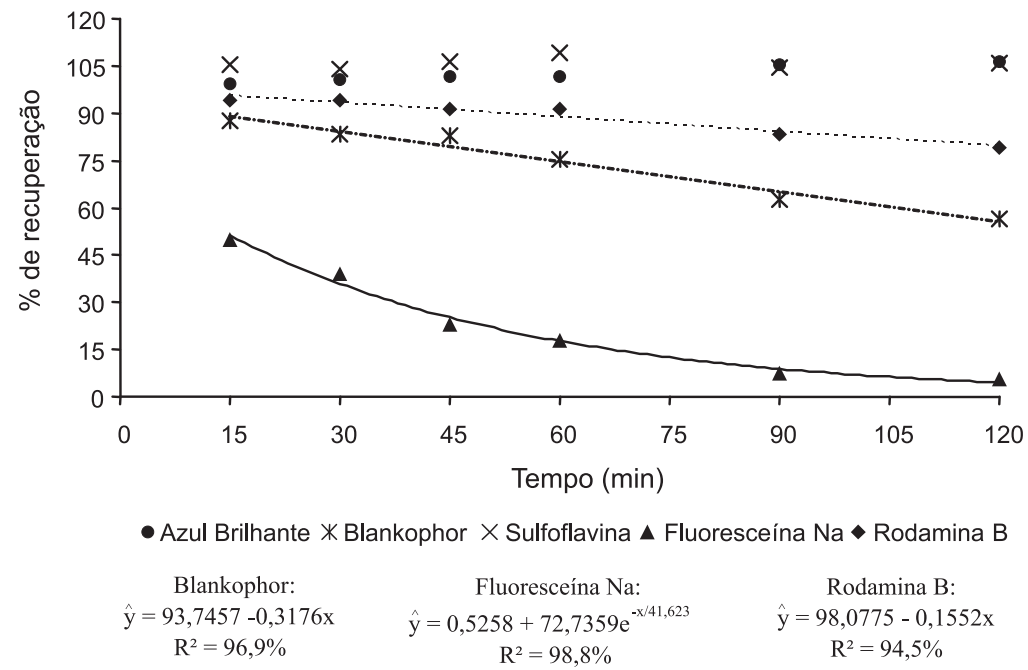

Figura 2 - Curvas de regressão para porcentagem de recuperação de traçadores expostos à luz solar.

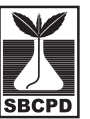

Planta Daninha, Viçosa-MG, v. 32, n. 4, p. 861-870, 2014 
período de até 300 minutos de exposição à luz solar. Brusselman et al. (2004) também concluíram que a BSF possui baixa fotodegradação em experimentos de campo.

Na Tabela 5 é mostrada a porcentagem de recuperação dos traçadores após submetidos à agitação. Observa-se que a BSF, a FSS e o $\mathrm{AB}$ demonstraram ser os mais estáveis à agitação, em todos os tempos, tendo valores acima de 95,4\%. A RDM igualou-se a estes traçadores apenas no tempo de 10 minutos de agitação, porém, em todos os tempos, teve porcentagem

Tabela 5 - Porcentagem de recuperação de traçadores após submetidos a diferentes tempos de agitação na rotação de $150 \mathrm{rpm}$

\begin{tabular}{|l|c|c|c|c|}
\hline \multirow{2}{*}{ Traçador } & \multicolumn{4}{|c|}{ Tempo de agitação (min) } \\
\cline { 2 - 5 } & 10 & 20 & 30 & 60 \\
\hline Rodamina B & $95,4 \mathrm{a}$ & $83,8 \mathrm{~b}$ & $88,8 \mathrm{~b}$ & $71,0 \mathrm{~b}$ \\
\hline Blankophor & $79,6 \mathrm{~b}$ & $75,7 \mathrm{c}$ & $69,7 \mathrm{c}$ & $63,5 \mathrm{c}$ \\
\hline Sulfoflavina & $100,2 \mathrm{a}$ & $98,3 \mathrm{a}$ & $100,0 \mathrm{a}$ & $95,4 \mathrm{a}$ \\
\hline Fluoresceína Na & $97,6 \mathrm{a}$ & $99,3 \mathrm{a}$ & $101,5 \mathrm{a}$ & $98,3 \mathrm{a}$ \\
\hline Azul Brilhante & $99,4 \mathrm{a}$ & $99,5 \mathrm{a}$ & $99,2 \mathrm{a}$ & $99,9 \mathrm{a}$ \\
\hline \multicolumn{3}{|c|}{$\mathrm{CV}=1,95 \%$} & \multicolumn{3}{c|}{$\begin{array}{c}\text { Ftraç }=203,088^{* *} \\
\text { Ftemp }=25,493^{* *} \\
\text { Finter }=8,726^{* *}\end{array}$} \\
\hline
\end{tabular}

Médias seguidas por letras distintas nas colunas diferem entre si pelo teste de Tukey a 0,05 de significância. CV: coeficiente de variação. $F_{\text {traç}}, F_{\text {temp }}, F_{\text {inter }}$ - valores de $F$ calculado para os fatores traçador, tempo e interação entre os fatores, respectivamente. ** significativo a 0,$01 ; *$ significativo a 0,05 . de recuperação maior do que a do BKP, cujos valores foram abaixo de $79,6 \%$.

O tempo de agitação para a BSF, a FSS e o $A B$ não interferiu na porcentagem de recuperação, o que reafirma que esses traçadores foram os mais estáveis à agitação (Figura 3). Entretanto, quanto maior o tempo de agitação, menor foi a porcentagem de recuperação para a RDM e o BKP. No caso da RDM, para cada 10 min de agitação a $150 \mathrm{rpm}$, espera-se redução de 4,4\% na porcentagem de recuperação, enquanto para o BKP espera-se queda menor: $3,2 \%$.

Uma utilização prática dessa análise é que, em aplicações cuja calda contendo o traçador seja exposta por um longo período de agitação constante no tanque do pulverizador, devemse evitar os traçadores RDM e BKP. Nesse caso, as melhores opções são a BSF, a FSS e o $\mathrm{AB}$. Contudo, convém ressaltar que a RDM mostrou boa estabilidade inicial, podendo ser utilizada, desde que o tempo de agitação seja menor do que 10 minutos. Além disso, ao se fazer a coleta da calda para medir a concentração do traçador, deve-se retirar a calda na saída da ponta de pulverização, a fim de reduzir as influências sobre a concentração do traçador provenientes da turbulência gerada pelo sistema hidráulico do pulverizador.

É importante salientar que não há um valor exato sobre a real condição a que a calda

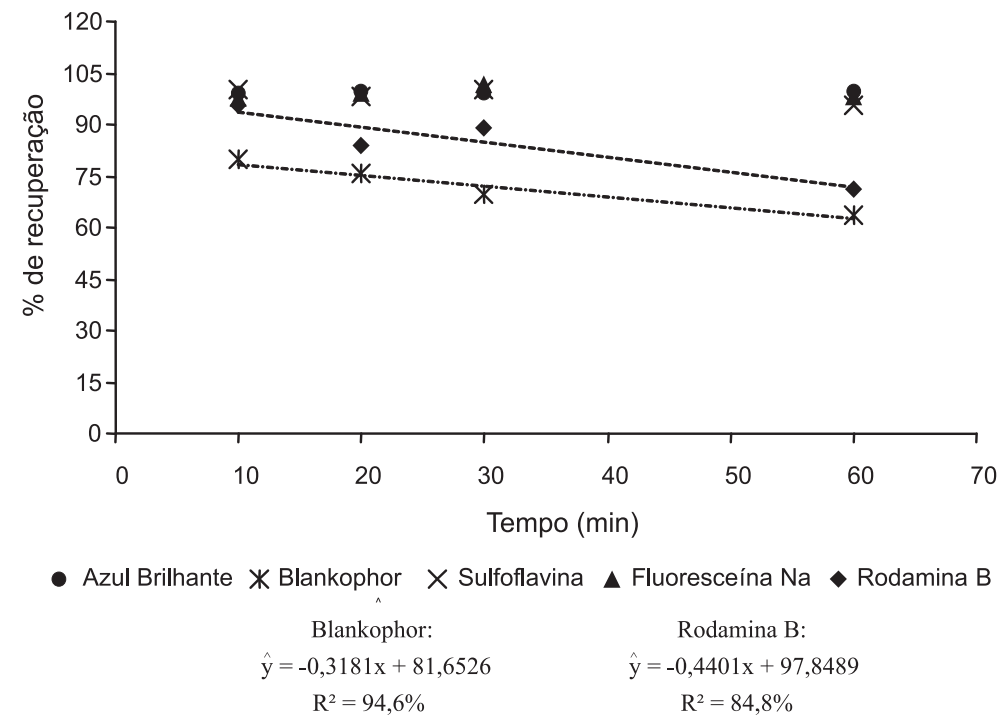

Figura 3 - Curvas de regressão para a porcentagem de recuperação de traçadores submetidos a diferentes tempos de agitação na rotação de $150 \mathrm{rpm}$. 
é submetida dentro do tanque de pulverização. Além disso, existem vários mecanismos de agitação, podendo ser pelo fluxo de retorno do líquido ou por pás agitadoras. Assim, novas pesquisas são necessárias para informar o grau de agitação a que a calda é submetida, o que, aliado ao equipamento utilizado na aplicação, pode permitir avaliar a real influência da agitação na quantificação dos traçadores.

Outro ponto refere-se à técnica de extração. As metodologias para extração dos traçadores RDM, BSF, AB e FSS envolvem a agitação, sobretudo quando há papéis como alvos. O maior cuidado deve ser dado à RDM, uma vez que os demais não sofreram influência. Portanto, deve-se extrair esse traçador utilizando-se rotações inferiores a $150 \mathrm{rpm}$, conforme metodologia descrita por Scramin et al. (2002), que propuseram a rotação de $120 \mathrm{rpm}$. No caso do BKP, extraí-lo de alvo de papel sem agitação, conforme proposto por Villalba et al. (2011), pode ser explicado pela perda gradual na fluorescência resultante da turbulência, indicando que, de fato, a extração deve ser feita com agitação manual ou em baixas rotações.

Conclui-se que a rodamina $B$ e a sulfoflavina brilhante foram os únicos traçadores que reduziram a tensão superficial da calda em relação à água destilada. A menor tensão superficial foi observada para a rodamina $\mathrm{B}: 66,17 \mathrm{mN} \mathrm{m}^{-1}$.

Todos os traçadores diminuíram o potencial hidrogeniônico da calda em relação à água destilada. O blankophor e a fluoresceína sódica foram os traçadores cuja calda teve o $\mathrm{pH}$ mais próximo do da água destilada. O menor $\mathrm{pH}$ da calda foi obtido pela sulfoflavina brilhante: 4,31.

Os traçadores não modificaram a viscosidade da água destilada, que foi de 1,25 mPa s.

A maior eficiência de extração em alvo de papel foi obtida pela sulfoflavina brilhante, seguida pela fluoresceína sódica e rodamina B.

A sulfoflavina brilhante e o azul brilhante demonstraram ser os traçadores mais estáveis à luz solar, e a fluoresceína sódica, o que mais se degradou após a exposição.

O blankophor foi o traçador que teve menor estabilidade à agitação da calda.
A sulfoflavina brilhante mostrou ser o traçador mais adequado aos estudos de deposição de calda e deriva nas aplicações de produtos fitossanitários.

Entre os traçadores de baixo custo e maior disponibilidade no Brasil, o corante fluorescente rodamina B mostrou ser a melhor opção ao se utilizar como alvo papel filtrante.

\section{AGRADECIMENTOS}

À Empresa Brasileira de Pesquisa Agropecuária (Embrapa), à Fundação de Amparo à Pesquisa do Estado de Minas Gerais (Fapemig) e ao Conselho Nacional de Desenvolvimento Científico e Tecnológico (CNPq), pelo apoio financeiro.

\section{LITERATURA CITADA}

BRUSSELMAN, E. et al. Wind tunnel tests with different tracer and collection techniques for the measurement of spray drift. Comm. Agric. Appl. Biol. Sci., v. 69, n. 4, p. 829-836, 2004.

CHAIM, A. et al. Deposição de agrotóxicos pulverizados na cultura da maçã. Pesq. Agropec. Bras., v. 38, n. 7, p. 889-892, 2003.

CUNHA, J. P. A. R.; ALVES, G. S. Características físicoquímicas de soluções aquosas com adjuvantes de uso agrícola. Interciência, v. 39, n. 4, p. 665-659, 2010.

De MARCHI, S. B. et al. Effect of spray tips and mix deposition on common water hyacinth growing with varied population arrangements of Salvinia and water lettuce.

J. Aquatic Plant Manag., v. 47, n. 2, p. 101-115, 2009.

De SCHAMPHELEIRE, M. et al. Deposition of spray drift behind border structures. Crop Protec., v. 28, n. 12, p. 1061-1075, 2009.

FERREIRA, D. F. Sisvar: um programa para análises e ensino de estatística. R. Symp., v. 6, n. 1, p. 36-41, 2008.

FRITZ, B. K. et al. Fluorescent tracer method for evaluating spray transport and fate of field and laboratory spray applications. J. ASTM Intern., v. 8, n. 3, p. 1-9, 2011.

GOUZIE, D. et al. Dye-tracing studies in southwestern Missouri, USA: indication of stratigraphic flow control in the Burlington Limestone. Hydrogeol. J., v. 18, n. 4, p. 1043-1052, 2010.

HAYDEN, J. et al. Two water-soluble optically resolvable dyes for comparing pesticide spray distribution. J. Econ. Entomol., v. 83, n. 6, p. 2411-2413, 1990. 
INMET. Consulta de dados estação automática: Uberlândia (MG). Disponível em: <http://www.inmet.gov.br/ sonabra/pg_dspDadosCodigo.php?QTUwNw==>. Acesso em: 18 fev. 2013.

MAGDALENA, J. C. Efecto de la utilización de pulverizadores de flujo transversal e hidropneumático tradicional sobre la calidad de los tratamientos fitosanitarios en manzanos (Malus domestica, Borkh). 2004. 144 f. Tese (Doutorado em Agronomia) Departamento de Mecanización y Tecnología Agraria, Valência, 2004.

MARCHI, S. R. et al. Degradação luminosa e retenção foliar dos corantes Azul Brilhante FDC-1 e Amarelo Tartrasina FDC-5 utilizados como traçadores em pulverizações.

Planta Daninha, v. 23, n. 2, p. 287-294, 2005.

MATUO, T. Desenvolvimento de um pulverizador intermitente operado fotoeletricamente para tratamento de pomares de citros. 1988. 167 f. Tese (Livre Docência) UNESP/FCAV, Jaboticabal, 1988.

NUYTTENS, D. et al. The influence of operator controlled variables on spray drift from field crop sprayers.

Trans. ASABE, v. 50, n. 4, p. 1129-1140, 2007.

PALLADINI, L. A. et al. Choice of tracers for the evaluation of spray deposits. Sci. Agríc., v. 62, n. 5, p. 440-445, 2005.

PERGHER, G. Recovery rate of tracer dyes used for spray deposit assessment. Trans. ASAE, v. 44, n. 4, p. 787-794, 2001.

PINTO, J. R. et al. Estabilidade à exposição solar dos traçantes azul brilhante e amarelo tartrasina utilizados em estudos de deposição de pulverização. R. Bras. Agroci., v. 13, n. 1, p. 105-107, 2007.
REZENDE, D. T. Análise comparativa de métodos para quantificação de depósitos da pulverização em diferentes alvos. 2011. 67 f. Dissertação (Mestrado em Agronomia) Faculdade de Ciências Agronômicas, Universidade Estadual Paulista "Júlio de Mesquita Filho", Botucatu, SP, 2011.

SCHLEIER, J. J. et al. The effect of fluorescent tracers on droplet spectrum, viscosity and density of pesticides formulations. J. Environ. Sci. Health, v. 45, n. 7 , p. 621-625, 2010.

SCRAMIN, S. et al. Avaliação de bicos de pulverização de agrotóxicos na cultura do algodão. R. Ecotoxicol. Meio Amb., v. 12, p. $43-50,2002$.

SILVA, F. A. S.; AZEVEDO, C. A. V. Principal componentys analyses in the software Assistat Statistical attendance. In: WORLD CONGRESS ON COMPUTER IN AGRICULTURE, 7., Reno, 2009. Anais... Reno: American Society of Agricultural and Biological Engineers, 2009.

SPSS Inc. SPSS statistics for Windows, version 17.0. Chicago: SPSS Inc, 2008.

SPSS Inc. Table curve 2D - automated curve fitting and equation discovery. Chicago: SPSS Science, 2000.

SUHOGUSOFF, A. V. et al. Adsorção do traçador fluorescente uranina em sedimentos quaternários da bacia de São Paulo. R. Bras. Geoci., v. 35, n. 4, p. 551-558, 2005.

VILLALBA, J. et al. Evaluación de la deposición de boquillas de aplicación para el control de Fusariosis em trigo.

Agrociência, v. 15, n. 2, p. 69-75, 2011.

ZHU, H. et al. Effect of solution $\mathrm{pH}$ conditions on fluorescence of spray deposition tracers. Trans. ASAE, v. 21, n. 3, p. 325-329, 2005. 